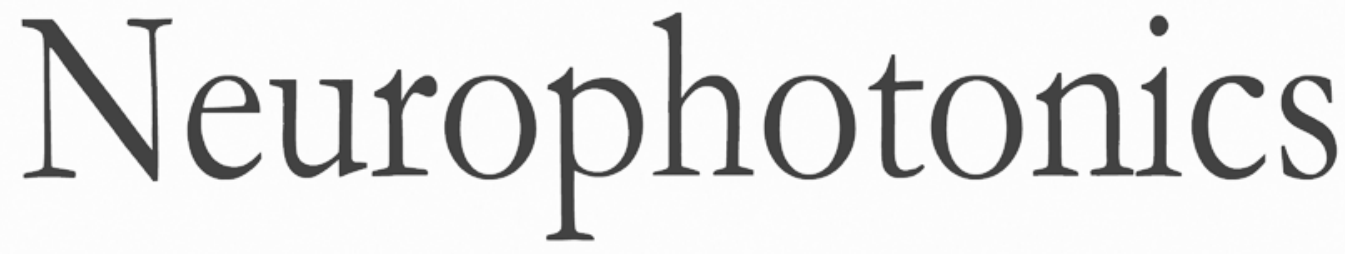

\title{
Distinguishing cognitive impairment by using singularity spectrum and lacunarity analysis of the retinal vascular network
}

Edmund Arthur

Gabor Mark Somfai

Maja Kostic

Susel Oropesa

Carlos Mendoza Santiesteban

Delia Cabrera DeBuc 


\title{
Distinguishing cognitive impairment by using singularity spectrum and lacunarity analysis of the retinal vascular network
}

\author{
Edmund Arthur, ${ }^{\text {a }}$ Gabor Mark Somfai, ${ }^{b, c}$ Maja Kostic, ${ }^{a}$ Susel Oropesa, ${ }^{a}$ Carlos Mendoza Santiesteban, ${ }^{a}$ \\ and Delia Cabrera DeBuc ${ }^{a}$ \\ aUniversity of Miami, Bascom Palmer Eye Institute, Department of Ophthalmology, Miami, Florida, United States \\ ${ }^{\mathrm{b}}$ Pallas Kliniken, Retinology Unit, Olten, Switzerland \\ 'Semmelweis University, Department of Ophthalmology, Budapest, Hungary
}

\begin{abstract}
The development of effective therapies for cognitive impairment (Cl), especially due to Alzheimer's disease, demands diagnosing the condition during the prodromal phase. The diagnosis of $\mathrm{Cl}$ involves expensive and invasive methods, such as positron emission tomography and cerebrospinal fluid assessment via spinal tap. Hence, a comparatively lower cost and noninvasive method of diagnosis is imperative. The human retina is an extension of the brain characterized by similarities in vascular and neural structures. The complications of $\mathrm{Cl}$ are not only limited to the brain but also affect the retina for which the loss of retinal ganglion cells has been associated with neurodegeneration in the brain. The loss of retinal ganglion cells in individuals with $\mathrm{Cl}$ may be related to reduced vascular demand and a potential remodeling of the retinal vascular branching complexity. Retinal imaging biomarkers may provide a low cost and noninvasive alternative for the diagnosis of $\mathrm{Cl}$. In this study, the retinal vascular branching complexity of patients with $\mathrm{Cl}$ was characterized using the singularity spectrum multifractal dimension and lacunarity parameter. A reduced vascular branching complexity was observed in subjects with $\mathrm{Cl}$ when compared to age- and sex-matched cognitively healthy controls. Significant associations were also found between retinal vascular and functional parameters. $\odot$ The Authors. Published by SPIE under a Creative Commons Attribution 4.0 Unported License. Distribution or reproduction of this work in whole or in part requires full attribution of the original publication, including its DOI. [DOI: 10.1117/1.NPh.6.4.041109]
\end{abstract}

Keywords: cognitive impairment; singularity spectrum; lacunarity; retinal vascular complexity; multifractal analysis.

Paper 19050SSR received May 24, 2019; accepted for publication Aug. 27, 2019; published online Sep. $23,2019$.

\section{Introduction}

There are approximately 46 million dementia patients worldwide according to the 2015 World Alzheimer Report. ${ }^{1}$ This number is likely to double every 20 years, and a projected increase to 131.5 million people by 2050 is expected. ${ }^{1}$ Alzheimer's disease (AD) is the most common, progressive cause of dementia in the elderly, and a severe burden on the aging society worldwide. ${ }^{1}$ As one of the top ten leading causes of death in North America, AD has no proven preventive or curative interventions. ${ }^{2}$ Early- and cost-effective diagnosis is critical to the next stage of treatment and drug development. ${ }^{2}$ However, progress in the management and treatment of $\mathrm{AD}$ is limited by constraints surrounding the early diagnosis of the disease. ${ }^{3-8}$ Although there are antiamyloid drugs that can slow down the accumulation of amyloid plaques in the brain and hence the progression of the disease, a difficulty lies in the detection of the disease during the prodromal phase. The accumulation of amyloid plaques in the brain is detected by expensive and invasive methods, such as positron emission tomography (PET) and cerebrospinal fluid assessment via a spinal tap. ${ }^{2}$ Therefore, a comparatively lower cost and noninvasive method may aid in the diagnosis of the disease.

The human retina is an extension of the brain. ${ }^{9}$ The retinal ganglion cells are similar to that of the cerebral cortex neurons, and the cerebral small vessels are similar to that of the retinal

*Address all correspondence to Delia Cabrera DeBuc, E-mail: dcabrera2 @ med miami.edu vessels. ${ }^{9}$ Also, it is well established that complications of cognitive impairment (CI) are not only limited to the brain but also affect the retina, which is evident in the neurodegeneration in the brain concomitant with the loss of retinal ganglion cells ${ }^{10,11}$ and reduced bioelectrical activity of the retinal neurons. ${ }^{12,13}$ Compared with standard neuroimaging techniques, imaging the retina is noninvasive and can be low cost. Therefore, the human retina may serve as a noninvasive window to study and possibly diagnose CI early during the prodromal phase of the disease. The neurodegeneration that occurs in the retina of cognitively impaired individuals may indicate reduced oxygen and nutrient demand as well as a potential remodeling of the retinal vascular network or branching pattern.

The human retinal vascular network holds a self-similarity or scaling property with a characteristic branching pattern. ${ }^{14-19}$ Therefore, the retinal vascular network produces similar patterns of different sizes at different magnification or scale. ${ }^{12,14-19}$ This self-similar property of the human retinal vascular network can be characterized by using a mathematical computational method termed fractal dimension (FD) analysis. A retinal vascular network with a more complex branching pattern has a large FD and vice versa. The retinal vascular branching pattern in patients with CI, ${ }^{12,20,21}$ diabetes, ${ }^{22-26}$ and amblyopia ${ }^{27}$ has been investigated using FD analysis with the goal of introducing an automated diagnostic approach for these specific conditions.

The calculation of the FD of the retinal vascular network comprises the skeletonization of images of the vessels obtained with a fundus camera or a scanning laser ophthalmoscope (SLO). These images are fitted with rectangular grids consisting 
of boxes of varying sizes. ${ }^{12,14-19}$ The slope of the regression line of the double log plot of the number of boxes versus the box side length is termed $D_{0}$ - the monofractal box-counting FD, which is a global measure that summarizes the whole branching pattern of the retinal vascular tree. ${ }^{14}$ The box-counting FD has been used to characterize the retinal vascular branching complexity in patients with $\mathrm{CI}^{21,28-30}$ However, the retinal vascular network has a multifractal characteristic rather than a monofractal pattern. Also, it is characterized by a hierarchy of scales rather than a single scale. ${ }^{17,23,25,27}$ Moreover, the multifractal behavior of the retinal vascular network is characterized by the generalized dimension spectrum $(D q$ versus $q)$ and the singularity spectrum $[f(\alpha)$ versus $\alpha] .^{17,23,25}$ In our previous study, the capacity or box-counting dimension $\left(D_{0}\right)$, the information dimension $\left(D_{1}\right)$, and the correlation dimension $\left(D_{2}\right)$ were reduced in the cognitively impaired participants compared to the cognitively healthy controls. $^{12}$

The lacunarity $(\Lambda)$ parameter is another metric used in the analysis of the retinal vascular network to measure the gap distribution or dispersion of the pixels within the retinal image. ${ }^{25,27,31}$ It characterizes the heterogeneity of the pixels within an image with a larger $\Lambda$, indicating a larger heterogeneity and vice versa. ${ }^{27}$ Also, it can distinguish between structures of similar FD. ${ }^{27,31}$ The multi-FD and $\Lambda$ parameter can both be combined to assess the retinal vascular network of cognitively impaired participants versus age- and sex-matched cognitively healthy controls as initial steps toward an automated diagnostic approach for this condition.

The purpose of this study is twofold. First, we compared the singularity spectrum and $\Lambda$ parameter of the retinal vascular network of patients with CI to age- and sex-matched cognitively healthy controls. We hypothesized that the singularity spectrum exponents will be reduced in cognitively impaired patients compared to cognitively healthy controls, indicating a less complex retinal branching pattern in the patients with CI. The $\Lambda$ parameter, on the other hand, was expected to be more heterogeneous or larger in the participants with CI compared to cognitively healthy controls. Second, we investigated the associations between retinal vascular parameters (i.e., singularity spectrum exponents and $\Lambda$ parameter) and functional parameters, such as the implicit time (IT) and amplitude of the electroretinogram (ERG), and the montreal cognitive assessment (MoCA) scores. These associations were hypothesized to be significant. The outcome of this study may serve as an initial step toward an automated diagnostic approach for the diagnosis of CI at low cost with the added value of an eye-screening.

\section{Materials and Methods}

\subsection{Study Participants}

Participants with CI were identified in a nonsystematic fashion as they appeared in the clinic or from a group of patients attending adult care centers and community clinics. Exclusion criteria included participants under the age of 55 years, history of any ophthalmic disease except for cataract surgery. The macular and optic disk regions of the fundus images of these subjects were examined for glaucoma, age-related macular degeneration, and other comorbid conditions and participants with such conditions were excluded from the study. Both hypertension and diabetes mellitus, as well as cardiovascular disease, were considered comorbid medical conditions related to retinal vascular alterations. In addition, current or history of study subject-reported smoking was considered in the study. All subjects underwent a cognitive function assessment with the MoCA test, ERG evaluation, as well as fundus imaging. Informed consent was obtained from all participants and for those whose CI affected their decision making on participation in the study, and an informed consent was obtained through an eligible proxy. The study adhered to the tenets of the declaration of Helsinki and was approved by the Human Research Ethics Committee of the University of Miami.

\subsection{Fundus Imaging and Skeletonization of Retinal Vessels}

The analysis of the retinal fundus images and skeletonization of the retinal vessels of the subjects in this study have been described in detail in our previous study. ${ }^{12}$ Briefly, optic disk centered images (FOV of $45 \mathrm{deg}$ ) of size $1024 \times 1024$ pixels of both eyes were taken with a nonmydriatic SLO digital camera (EasyScan, iOptics, the Netherlands). To ensure the independence of samples, either eye of each subject was chosen based on good image scan quality index. The retinal vessels in the entire $45 \mathrm{deg}$ images were skeletonized, as described in our previous study. ${ }^{12}$ This method of skeletonization provides more samples of vessels for further analysis unlike using only a select few vessels around the optic disk, as has been done previously. ${ }^{20,21,32}$

\subsection{ERG and Cognitive Function Assessment}

The assessment of the bioelectrical activity of the retina was performed with a full-field ERG (RETeval ${ }^{\mathrm{TM}}$, LKC Technologies, Inc., Gaithersburg, Maryland, United States) based on the International Society for Clinical Electrophysiology of Vision (ISCEV) protocol. ${ }^{12,33-35}$ The ISCEV 6 protocol was used, which first includes a light-adapted state, consisting of lightadapted ERG (stimulus strength, $3.0 \mathrm{~cd} . \mathrm{s} / \mathrm{m}^{2}$; frequency, $28.3 \mathrm{~Hz}$ flicker response) and dark-adapted ERG, including rod, maximal dark-adapted, and cone responses. ERG IT and amplitudes were elicited by 141 to 424 flashes separately for each eye.

$\mathrm{CI}$ is a condition that individuals face when they have trouble remembering, learning new things, concentrating, or making decisions that affect their everyday life. Therefore, assessment of the cognitive function of study participants was conducted using the MoCA test to identify CI. ${ }^{12}$ This test is a one-page 30 -point assessment performed in $\sim 10$ to $12 \mathrm{~min}$ with a score range of 0 to 30 . Typical scores $<26$ indicate some form of CI. ${ }^{36,37}$ Detailed assessment of CI using MoCA as well as the MoCA scores of the participants in our current study have been provided previously. ${ }^{12}$

\subsection{Singularity Spectrum by Multifractal Analysis}

The network of blood vessels of the human retina holds a fractal structure with a vascular branching process characterized by self-similarity and scaling. The FD analysis is a computational method that characterizes complexity in a natural phenomenon and has been extensively used to characterize the branching complexity of the human retinal vasculature..$^{12,17,23-27,38}$ The fractal analysis of the human retinal vasculature measures the retinal vascular branching pattern with a complex branching pattern indicating a larger FD and vice versa. This method has been extensively used in the characterization of the retinal vascular network in patients with $\mathrm{CI},{ }^{12,20,21}$ diabetes, ${ }^{22-26}$ and amblyopia. $^{27}$ 
Self-similarity over varying scales or magnification is an important characteristic of fractal structures. The concept is that at different magnification or scales, a fractal structure exhibits a similar pattern at different sizes. ${ }^{12,14-19}$ This characteristic of fractal structures can be described by the equation: ${ }^{12}$

$N(r)=$ const $r^{-D}$,

where $N(r)$ refers to certain measurements applied on the complex structure of the fractal object at varying magnification or scaling $r$. The parameter $D$ is the FD metric, which refers to the number of self-similar patterns generated from the fractal object as the magnification or scale $r$ increases or decreases. ${ }^{12,14-19}$ The FD calculated with the box-counting method is a common monofractal type of FD that used to characterize the human retinal vascular complexity. ${ }^{20-22,26}$ In this method, the segmented or skeletonized retinal vessels from a retinal fundus or SLO camera are fitted with a rectangular grid consisting of large amount of boxes. ${ }^{12,26}$ The number of boxes in the rectangular grid in this scenario is $N(r)$ with a box having side length $r$, as shown in Eq. (1). Once a double log plot of the number of boxes $N(r)$ and the box side length $r$ are obtained, the slope of the regression line in this plot indicates the monofractal FD (box counting-based) metric termed $D_{0}$, as shown in Eq. (2): ${ }^{12,26}$

$D_{0}=\lim _{r \rightarrow 0} \log N r / \log \left(\frac{1}{r}\right)$.

Because the retinal vessel network geometry has a finer texture or heterogeneity in its space-filling characteristics, its morphological property cannot be described sufficiently by a global parameter reflecting a simple FD obtained by monofractal analysis. Therefore, the human retinal vascular network is considered a geometric multifractal structure or a set of intertwined fractals characterized by a hierarchy of exponents rather than a single FD as in $D_{0}$ (i.e, when the retinal vascular network is considered to have a monofractal pattern). Therefore, the multifractal property of the human retinal vascular network is characterized by the generalized dimension spectrum $(D q$ versus $q$, where $D q$ represents the FD at the $q$ 'th order or exponent) and the singularity spectrum $[f(\alpha)$ versus $\alpha] \cdot{ }^{17,25,27}$ The singularity spectrum of the FD $f(\alpha)$ versus the singularity exponent $\alpha$ is defined as ${ }^{17,25}$

$N(\alpha)=r^{-f(\alpha)}$,

where $N(\alpha)$ is the number of boxes, such that the probability $P_{i}$ $(r)$ of finding a pixel within a given region i scales, as observed in Eq. (4): $:^{17,25}$

$P_{i}=r^{\alpha i}$

$f(\alpha)$ is hence the FD of all the regions with singularity strengths between $\alpha$ and $\alpha+d \alpha$, where the singularity exponent $\alpha$ takes on values within the interval $-\infty$ and $+\infty$.

The relationship between the $D(q)$ spectrum and the $f(\alpha)$ spectrum is established through the Legendre transformation, as shown in Eq. (5): ${ }^{17-19}$

$f(\alpha(q))=q \alpha(q)-\tau(q)$ where $\alpha(q)$ represents the singularity exponent at the $q$ 'th order moment expressed ${ }^{17-19}$

$\alpha(q)=d \tau(q) / d q$

and $\tau(q)$ represents the mass correlation exponent of the $q$ 'th order related to $D q$, as observed in Eq. (7): ${ }^{17-19}$

$\tau(q)=(q-1) D q$

where for $q=1$ and $\tau(1)=0$.

The singularity FD $[f(\alpha(q))]$ and exponent $\alpha(q)$ of the $q$ 'th order can be computed using the software Image J (Wayne Rasband, National Institutes of Health in Bethesda, Maryland, United States) together with the FracLac plugin (A. KarperienCharles Sturt University, Australia) ${ }^{39}$ with the settings, as previously described. ${ }^{12}$ Hence, $q$ represents values from -10 to +10 with an increment of 1 . These values are computed and then averaged with their standard deviations at each value of $q$. Then, a plot of $f[\alpha(q)]$ versus $\alpha(q)$ with standard deviation error bars is obtained, representing the singularity spectrum. ${ }^{17,25}$ For a multifractal object, the singularity spectrum is typically a parabola with concavity facing down. ${ }^{17,23,25}$

Typically, the height $(\Delta f)$, width $(\Delta \alpha)$, and asymmetry $(A)$ of the parabola are the measures used to describe the singularity spectrum, as shown in Eqs. (8)-(10) below. ${ }^{23,25,40}$ The $\alpha$ values $\left(\alpha_{0}, \alpha_{1}\right.$, and $\left.\alpha_{2}\right)$ of the singularity spectrum at $q=0,1,2$ can also be compared between groups with greater $\alpha$ values indicating greater singularities or maxima and vice versa: ${ }^{17}$

$\Delta f(\alpha)=f(\alpha)_{\min }-f(\alpha)_{\max }$,

where $f(\alpha)_{\max }$ and $f(\alpha)_{\min }$ represent the maximum and minimum $f(\alpha)$ values, respectively, ${ }^{23,40}$

$\Delta \alpha=\alpha_{\max }-\alpha_{\min }$.

Also, $\alpha_{\max }$ and $\alpha_{\min }$ represent the maximum and minimum $\alpha$ values, respectively. ${ }^{25}$ The higher the $\Delta \alpha$, the stronger is the multifractality, and the more complex is the pixel distribution within the image: ${ }^{25,41,42}$

$A=\left(\alpha_{0}-\alpha_{\min }\right) /\left(\alpha_{\max }-\alpha_{0}\right)$.

The parabola of the singularity or $F$ spectrum is symmetric when $A=1$, left skewed when $A>1$, and right skewed when $A<1 .^{23,25}$ A left-skewed $F$ spectrum means that there is a stronger presence of high fractal exponents and a significant fluctuation, while a right-skewed $F$ spectrum, on the other hand, posits low fractal exponents and a slight fluctuation. ${ }^{25}$

\subsection{Lacunarity Analysis}

The $\Lambda$ parameter measures the gap dispersion or the heterogeneity within an object. ${ }^{25,27,31}$ It can distinguish between two objects with similar fractality and it is a parameter that describes the coarseness or texture of an image. ${ }^{27,31}$ The $\Lambda$ parameter of the skeletonized images of the participants was computed with the ImageJ software together with the FracLac plugin with the settings used expatiated, as previously described. ${ }^{12}$ The ImageJ software together with the FracLac plugin computes the $\Lambda$ parameter based on the variation in pixel density at different box 
sizes in fixed and sliding scans. A lower $\Lambda$ parameter indicates more homogeneity of the pixel distribution in the image and vice versa. ${ }^{27}$ The mean $\Lambda$ parameter computed from the Image J software together with the FracLac plugin is then summarized as shown in Eq. (11): $:^{25,27,31}$

$\Lambda=\frac{1}{n}\left(\sum_{j=1}^{g} \sum_{i=1}^{n}\left[1+\left(\frac{\sigma}{\mu}\right) 2\right]\right)$,

where $\sigma$ is the standard deviation of the number of pixels that were in a box of size $\varepsilon ; \mu$ is the mean for pixels per box at this size $\varepsilon$, in a box count at an orientation $g$; and then, $n$ is the number of box sizes. ${ }^{25,27}$

\subsection{Statistical Analysis}

All statistical analyses were performed using IBM SPSS Statistics for Windows, Version 24.0 (IBM Corporation, Armonk, New York, USA). All values are presented as mean $\pm \mathrm{SD}$. The Shapiro-Wilk test of normality was used to test the Gaussian distribution of $\alpha_{0}, \alpha_{1}, \alpha_{2}, \Delta \alpha, A, \Delta f \alpha$, and $\Lambda$. Independent sample $t$-test was used to compare these parameters between the cognitively impaired and cognitively healthy participants assuming Levene's test for equality of variance was not statistically significant. In cases where the Shapiro-Wilk test was significant for any of the parameters, the Mann-Whitney $U$-test was used to compare that parameter between the two groups. Cohen's $d$ was used as an effect size measure when a parameter was significant when compared between the two groups, calculated using GPower calculator 3.1. ${ }^{43}$ A Cohen's $d$ value of 0.2 was considered small, 0.5 was considered medium, and $\geq 0.8$ was considered large. ${ }^{44}$ Pearson productmoment correlation was used to assess the relationship between the retinal vascular parameters $\left(\alpha_{0}, \alpha_{1}, \alpha_{2}\right.$, and $\left.\Lambda\right)$ and functional parameters (ERG IT and amplitude, and MoCA) for the cognitively impaired participants with the value $\geq 0.7$ considered as high. A $p$ value $<0.05$ was considered statistically significant.

\section{Results}

A total of 20 individuals with CI and 19 cognitive healthy participants were involved in this study. The average age of the CI participants ( $81 \pm 6$ years) did not significantly differ from that of the cognitively healthy participants ( $80 \pm 7$ years), $t(37)=0.38, p=0.70$. The proportion of males $(20 \%)$ and females $(80 \%)$ in the participants with CI did not significantly differ from the proportion of males (16\%) and females (84\%) in the cognitive healthy participants (Fisher's exact test, $p=1.00$ ). The singularity spectrum of both the cognitively impaired and cognitive healthy participants followed a parabola with concavity facing down demonstrating the multifractal property of the retinal vascular network of the two groups (Fig. 1). The singularity spectrum of the participants with CI also showed a trend or a shift toward lower singularity exponents or $\alpha$ range and had lower maxima compared to the singularity spectrum of the cognitively healthy participants even though there was quite an overlap for $\Delta f$ and $\Delta \alpha$ between the two groups [Fig. 1(c)].

The values of $\alpha_{0}, \alpha_{1}$, and $\alpha_{2}$ were significantly greater in the cognitively healthy participants than in the cognitively impaired participants, with greater than moderate Cohen's $d$ or effect size for $\alpha_{0}$ and $\alpha_{1}(d=0.63,0.63$, respectively $)$ and a large Cohen's $d$ for $\alpha_{2}(d=0.97)$ (Table 1). Thus, the participants with CI had lower singularity or $\alpha$ values compared to the cognitively
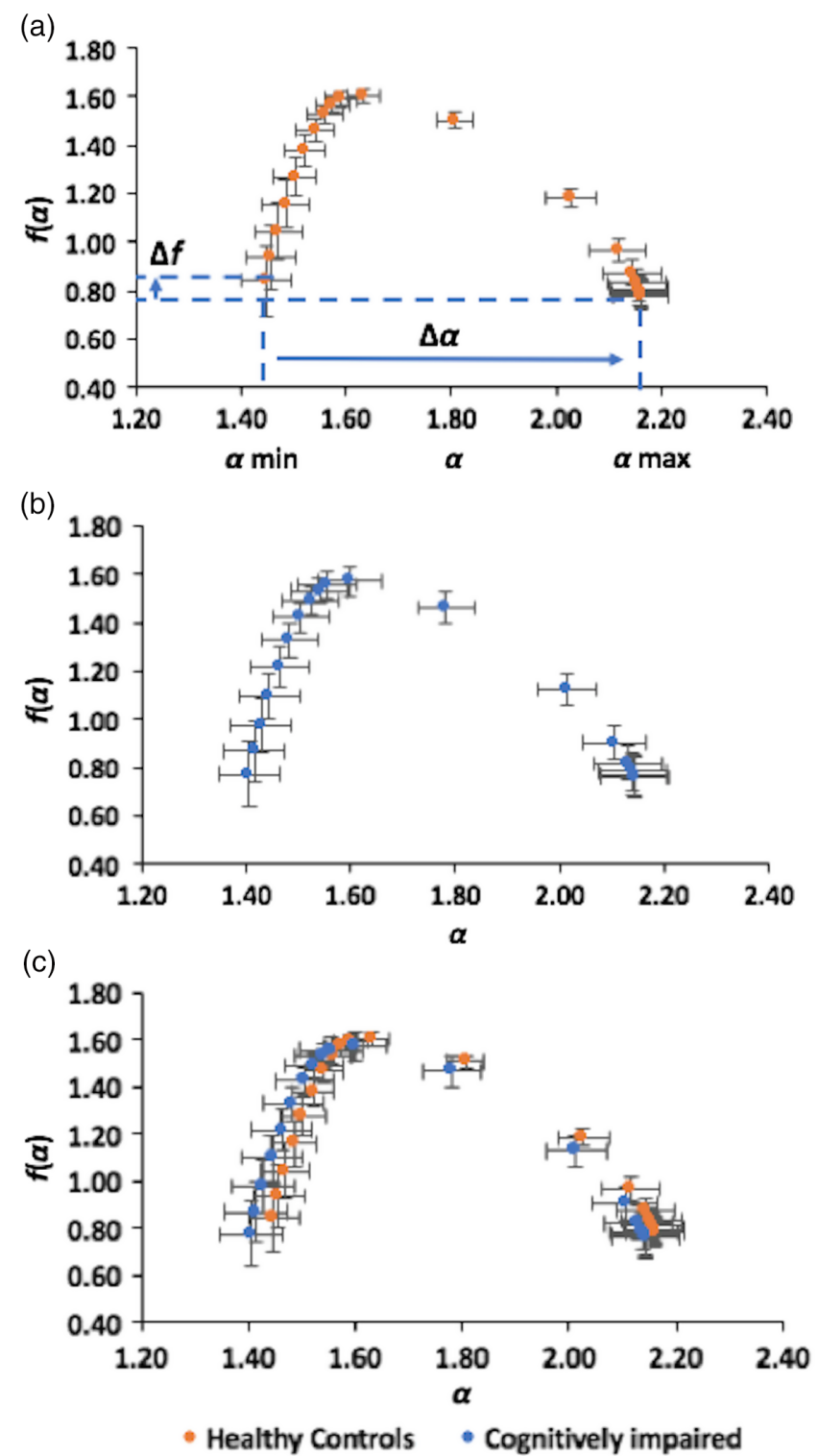

Fig. 1 Singularity or $F$ spectrum of cognitively impaired and cognitively healthy participants showing a parabola with concavity facing down demonstrating the multifractality of the retinal vessels of both groups. (a) The singularity spectrum of the cognitively healthy participants. The singularity spectrum shows a multifractal behavior, as demonstrated by the parabola with concavity facing down. (b) Singularity spectrum of the participants with $\mathrm{Cl}$ showing a multifractal behavior, as demonstrated by the parabola with concavity facing down. (c) Singularity spectrum of both groups showing a trend or shift of the singularity spectrum in participants with $\mathrm{Cl}$ toward a lower $\alpha$ range or maxima but quite an overlap of $\Delta f$ and $\Delta \alpha$ between the two groups. Error bars represent standard deviation.

healthy participants and this difference had a high effect size for $\alpha_{2}$ but not $\alpha_{0}$ and $\alpha_{1}$. The values of $\Delta f, \Delta \alpha$, and $A$ of the $F$ spectrum did not significantly differ between the cognitively healthy participants and the participants with CI (Table 1). The singularity spectrum of both the cognitively impaired and cognitively healthy participants was right-skewed $(A<1)$, indicating the presence of low fractal exponents and a slight fluctuation. Moreover, the $\Lambda$ parameter was not significantly different between the participants with CI and the cognitively healthy participants (Table 1). 
Table 1 Multifractal and Lacunarity parameters (mean \pm SD) obtained for the cognitively impaired and cognitive healthy participants.

\begin{tabular}{lcccc}
$\begin{array}{l}\text { Multifractal and } \\
\text { lacunarity } \\
\text { parameters }\end{array}$ & $\begin{array}{c}\text { Cognitively } \\
\text { impaired } \\
\text { group }\end{array}$ & $\begin{array}{c}\text { Cognitively } \\
\text { healthy } \\
\text { group }\end{array}$ & $p$ value & Cohen's $d$ \\
\hline$\alpha_{0}$ & $1.60 \pm 0.06$ & $1.63 \pm 0.03$ & 0.03 & 0.63 \\
$\alpha_{1}$ & $1.56 \pm 0.06$ & $1.59 \pm 0.03$ & 0.03 & 0.63 \\
$\alpha_{2}$ & $1.54 \pm 0.05$ & $1.58 \pm 0.03$ & 0.02 & 0.97 \\
$\Delta f$ & $0.87 \pm 0.10$ & $0.87 \pm 0.07$ & 0.93 & NA \\
$\Delta \alpha$ & $0.74 \pm 0.05$ & $0.71 \pm 0.05$ & 0.14 & NA \\
$A^{\text {a }}$ & $0.35 \pm 0.06$ & $0.35 \pm 0.05$ & 0.99 & NA \\
$\Lambda$ & $0.35 \pm 0.05$ & $0.34 \pm 0.03$ & 0.48 & NA \\
\hline
\end{tabular}

Note: $\alpha_{0}, \alpha_{1}$, and $\alpha_{2}$ represent the singularity exponents at $q=0,1,2$, respectively. The $\Delta f, \Delta \alpha$, and $A$ represent the height, width, and asymmetry of the singularity spectrum, respectively. The $\Lambda$ parameter represents lacunarity, indicating the gap dispersion within the image. NA: not applicable.

aMann-Whitney $U$-test was performed, otherwise an independent sample $t$-test was performed.

There was a significant positive association between retinal vascular singularity exponents $\left(\alpha_{0}, \alpha_{1}\right.$, and $\left.\alpha_{2}\right)$ and the IT, which was one of the functional measures obtained with the ERG assessments (Table 2). In particular, there was a high Pearson correlation coefficient between $\alpha_{2}$ versus ERG IT. However, a negative association was observed between the $\Lambda$ parameter versus the ERG IT (Table 2). There were no such associations between $\alpha_{0}, \alpha_{1}$, and $\alpha_{2}$, and $\Lambda$ versus the ERG amplitude and MoCA, $p>0.05$, Table 2 .

Table 2 Association between retinal vascular measures (i.e., multifractal and lacunarity parameters) and functional measures (ERG IT, ERG amplitude, and MoCA).

\begin{tabular}{lcc} 
Parameters & Pearson's correlation $(r)$ & $p$ value \\
\hline$\alpha_{0}$ versus ERG IT & 0.61 & 0.004 \\
$\alpha_{1}$ versus ERG IT & 0.67 & 0.001 \\
$\alpha_{2}$ versus ERG IT & 0.71 & $<0.001$ \\
$\Lambda$ versus ERG IT & -0.51 & 0.022 \\
$\alpha_{0}$ versus ERG amplitude & 0.41 & 0.07 \\
$\alpha_{1}$ versus ERG amplitude & 0.35 & 0.13 \\
$\alpha_{2}$ versus ERG amplitude & 0.29 & 0.22 \\
$\Lambda$ versus ERG amplitude & -0.23 & 0.33 \\
$\alpha_{0}$ versus MoCA & 0.43 & 0.06 \\
$\alpha_{1}$ versus MoCA & 0.43 & 0.06 \\
$\alpha_{2}$ versus MoCA & 0.42 & 0.07 \\
$\Lambda$ versus MoCA & -0.18 & 0.44 \\
\hline
\end{tabular}

\section{Discussion}

There is an emerging interest in the identification of retinal vascular and neural biomarkers for the early diagnosis of CI, especially due to $\mathrm{AD}$, during the prodromal phase of the disease because the neurodegeneration in the brain of $\mathrm{AD}$ patients occurs years before clinical symptoms appear. ${ }^{3-8,12,21,45-50}$ Although there is active neurodegeneration occurring in the brain, patients in the prodromal phase of the disease typically can go about their daily activities without showing any signs of CI. Therefore, the target of treatment and management to slow down disease progression is to diagnose the disease during the prodromal phase ${ }^{51}$ However, AD and other related dementia diseases are diagnosed through expensive and invasive methods, such as PET and cerebrospinal fluid assessment via a spinal tap. ${ }^{2}$ Therefore, there is a desire for a comparatively lower cost and noninvasive method that may aid in the early diagnosis of the disease. As the human retina is an extension of the brain and retinal imaging can be attained noninvasively at low cost, the human retina offers a noninvasive window to identify retinal vascular and neural biomarkers of brain diseases.

In this study, we identified changes in retinal vascular parameters (FD and $\Lambda$ ) in patients with CI that were significantly associated with a particular functional measure obtained with the ERG assessment (IT). Also, changes in some fractal exponents and their association with the bioelectrical activity of the retina (ERG IT) were found to have a large effect size. These associations interestingly pointed to a clear perturbation of the neurovascular component as a result of abnormal conditions mediated by the individual's disease status affecting both the brain and eye structures. We also found significantly reduced singularity spectrum exponents $\left(\alpha_{0}, \alpha_{1}\right.$, and $\left.\alpha_{2}\right)$ in the participants with CI compared to the cognitively healthy participants with a large effect size difference for $\alpha_{2}$ (Table 1), which may indicate that the reduced retinal vascular branching complexity for patients with CI, possibly due to reduced retinal neural demand, could be incorporated as a clinical tool for the diagnosis of CI. The multifractal behavior of the retinal vasculature of both groups is consistent with that found in previous studies. ${ }^{17,23,25}$ However, there was quite an overlap or no significant difference between the height $(\Delta f)$, width $(\Delta \alpha)$, and asymmetry $(A)$ of the singularity spectrum of both groups (Fig. 1 and Table 1). As in a previous study comparing patients with diabetic retinopathy and healthy controls, the lack of a significant difference in the width and asymmetry of the singularity spectrum as well as in the gap dispersion of the pixels $(\Lambda)$ could be attributed to the small sample size. ${ }^{25}$

Previous studies have investigated the fractal properties of the retinal vasculature of patients with CI. ${ }^{12,20,21}$ However, most studies that investigated the FD of cognitively impaired patients used the monofractal box-counting dimension approach rather than a multifractal spectrum methodology. ${ }^{20,21}$ Also, these studies have limited their analyses by sampling few vessels around the optic disk rather than using the whole skeletonized vessel tree in the entire fundus image of the participants. ${ }^{20,21} \mathrm{We}$ have previously characterized the multifractal behavior of the retinal vasculature of participants with CI versus cognitively healthy participants using only the generalized dimension spectrum. ${ }^{12}$ The results obtained for $\alpha_{0}, \alpha_{1}$, and $\alpha_{2}$ demonstrated that these singularity exponents were significantly reduced in the cognitively impaired individuals versus controls, which is consistent with the results of our previous study, where $D_{0}, D_{1}$, and $D_{2}$ were also significantly reduced in the cognitively imapired 
group. ${ }^{12}$ The significantly reduced FD found in the group with $\mathrm{CI}$ is also consistent with that found in other studies that investigated the retinal vascular branching pattern of cognitively impaired participants although a monofractal box-counting approach was used in these studies. ${ }^{20,21}$

The neurovascular coupling concept of the retina calls for increased vascular demand with associated increased neural activity ${ }^{52,53}$ It is well established that the neurodegeneration that occurs in the brain of patients with $\mathrm{CI}$ is associated with loss of retinal ganglion cells. ${ }^{10,11}$ This trend implies that there will be reduced vascular demand in terms of nutrients and oxygen from the retinal neurons in these subjects and a potential remodeling of the branching pattern complexity of the retinal vessels in these subjects. This tendency may explain the significantly reduced singularity spectrum exponents, as well as a trend toward lower maxima in the subjects with CI versus the ageand sex-matched cognitively healthy controls. Interestingly, this trend toward lower singularity spectrum exponents or maxima for the cognitively impaired participants is consistent with that found in another study that investigated the singularity spectrum for various retinal pathological conditions. ${ }^{17}$ The significant difference in the singularity exponents between the two groups had a high effect size for $\alpha_{2}$ despite the small sample size in our study. This particular result implies that the retinal vessel branching pattern of the participants with CI was less complex compared with the cognitively healthy participants and this difference had a large effect size beyond statistical significance. Therefore, the singularity exponents of the $F$ spectrum multifractal dimension could be incorporated into an automated diagnostic approach for participants with CI using the information of the retinal vascular branching complexity.

The significant associations between retinal vascular parameters and the ERG IT followed a positive trend for the retinal vascular FD parameters $\left(\alpha_{0}, \alpha_{1}\right.$, and $\left.\alpha_{2}\right)$ but a negative trend for $\Lambda$ (Table 2). Specifically, the negative association between the $\Lambda$ parameter and the ERG IT is expected as FD and $\Lambda$ are hypothesized to be negatively associated. ${ }^{54}$ The significant positive associations between the retinal vascular FD parameters $\left(\alpha_{0}, \alpha_{1}\right.$, and $\left.\alpha_{2}\right)$ and ERG IT had a large effect size or Pearson correlation coefficient for $\alpha_{2}$ (Table 2), which is consistent with the concept of neurovascular coupling. ${ }^{52,53}$ These associations are also consistent with the results of our previous study, which found significant positive associations between the generalized dimension spectrum and ERG IT. ${ }^{12}$

The results of this study should be interpreted in the light of some limitations. First, the sample size of our study was small, and this may have accounted for the overlap or lack of significant difference in the $\Lambda$ parameter, height, width, and asymmetry of singularity spectrum between the two groups as well as the lack of significant associations between the retinal vascular FD and the $\Lambda$ parameter versus the ERG amplitude and the MoCA scores for the cognitively impaired group. However, the sample size did not affect the significant difference and large effect size found for the retinal vascular FD parameters between the two groups as well as the associations between the retinal vascular and functional parameters. Second, the cause of CI for our patients is unknown-this could be due to $\mathrm{AD}$, vascular dementia, frontotemporal lesion, and other dementia-related diseases. The causation inquiry may require a more comprehensive approach, including the use of PET, magnetic resonance imaging, and cerebrospinal fluid analysis.
The process of finding biomarkers is complicated, and despite the small sample size of our study's population, we found significant associations between retinal vascular and functional parameters with large effect sizes that can be incorporated into longitudinal studies with a larger sample size for an automated diagnostic approach that could aid the detection of CI using retinal biomarkers. These multimodal parameters could be implemented in an automated screening setting using lowcost fundus imaging devices that could even be coupled with low-cost electrophysiological screening tools. All this together, used in an appropriate setting and targeting populations at high risk could, in turn, help not only obtain an early diagnosis, and thus a reduced burden on society, but also serve in the better understanding of the development of CI.

\section{Disclosures}

The University of Miami and Delia Cabrera DeBuc have filed a provisional patent application 62/776,693. The other authors report no conflict of interest and have no proprietary interest in any of the materials published in this article.

\section{Acknowledgments}

This study was supported in part by the Finker Frenkel Legacy Foundation, U.S. National Institutes of Health Center Grant No. P30-EY014801 to the University of Miami, an unrestricted grant to the University of Miami from Research to Prevent Blindness, Inc., and the Alzheimer's Association (Grant No. AARGD-17-531255, DCD).

\section{References}

1. M. J. Prince, "World Alzheimer Report 2015: the global impact of dementia: an analysis of prevalence, incidence, cost and trends," 2015, Alzheimer's Disease International, http:/www.alz.co.uk/ research/WorldAlzheimerReport2011.pdf (accessed 2 January 2019).

2. E. M. Lad et al., "Evaluation of inner retinal layers as biomarkers in mild cognitive impairment to moderate Alzheimer's disease," PLoS One 13(2), e0192646 (2018).

3. A. La Rue and L. F. Jarvik, "Cognitive function and prediction of dementia in old age," Int. J. Aging Hum. Dev. 25(2), 79-89 (1987).

4. R. T. Linn et al., "The 'preclinical phase' of probable Alzheimer's disease. A 13-year prospective study of the Framingham cohort," Arch. Neurol. 52(5), 485-490 (1995).

5. D. A. Snowdon et al., "Linguistic ability in early life and cognitive function and Alzheimer's disease in late life. Findings from the Nun Study," JAMA 275(7), 528-532 (1996).

6. H. Braak and E. Braak, "Frequency of stages of Alzheimer-related lesions in different age categories," Neurobiol. Aging 18(4), 351-357 (1997).

7. M. F. Elias, "The preclinical phase of alzheimer disease: a 22-year prospective study of the Framingham cohort," Arch. Neurol. 57(6), 808-813 (2000).

8. C. H. Kawas et al., "Visual memory predicts Alzheimer's disease more than a decade before diagnosis," Neurology 60(7), 1089-1093 (2003).

9. D. C. DeBuc, G. M. Somfai, and A. Koller, "Retinal microvascular network alterations: potential biomarkers of cerebrovascular and neural diseases," Am. J. Physiol. Heart. Circ. Physiol. 312(2), H201-H212 (2017).

10. B. Katz and S. Rimmer, "Ophthalmologic manifestations of Alzheimer's disease," Surv. Ophthalmol. 34(1), 31-43 (1989).

11. H. Hampel et al., "Revolution of Alzheimer precision neurology. Passageway of systems biology and neurophysiology," J. Alzheimer Dis. 64(s1), S47-S105 (2018).

12. D. C. DeBuc et al., "Investigating multimodal diagnostic eye biomarkers of cognitive impairment by measuring vascular and neurogenic changes in the retina," Front. Physiol. 9, 1721 (2018). 
13. M. M. Moschos et al., "Structural and functional impairment of the retina and optic nerve in Alzheimer's disease," Curr. Alzheimer Res. 9(7), 782-788 (2012).

14. G. Liew et al., "The retinal vasculature as a fractal: methodology, reliability, and relationship to blood pressure," Ophthalmology 115(11), 1951-1956.e1 (2008).

15. V. F. Cosatto et al., "Retinal vascular fractal dimension measurement and its influence from imaging variation: results of two segmentation methods," Curr. Eye Res. 35(9), 850-856 (2010).

16. G. N. Thomas et al., "Measurement of macular fractal dimension using a computer-assisted program," Invest. Ophthalmol. Visual Sci. 55, 2237-2243 (2014).

17. T. Stosic and B. D. Stosic, "Multifractal analysis of human retinal vessels," IEEE Trans. Med. Imaging 25(8), 1101-1107 (2006).

18. S. Talu, "Characterization of retinal vessel networks in human retinal imagery using quantitative descriptors," Hum. Vet. Med. 5(2), 52-57 (2013).

19. S. Talu, "Multifractal geometry in analysis and processing of digital retinal photographs for early diagnosis of human diabetic macular edema," Curr. Eye Res. 38(7), 781-792 (2013).

20. C. Y. Cheung et al., "Retinal vascular fractal dimension is associated with cognitive dysfunction," J. Stroke Cerebrovasc. Dis. 23(1), 43-50 (2014).

21. C. Y. Cheung et al., "Microvascular network alterations in the retina of patients with Alzheimer's disease," Alzheimer Dement. 10(2), 135-142 (2014).

22. M. Kostic et al., "Investigating the fractal dimension of the foveal microvasculature in relation to the morphology of the foveal avascular zone and to the macular circulation in patients with Type 2 diabetes mellitus," Front. Physiol. 9, 1233 (2018).

23. T. Zhu et al., "Multifractal and lacunarity analyses of microvascular morphology in eyes with diabetic retinopathy: a projection artifact resolved optical coherence tomography angiography study," Microcirculation 26(3), e12519 (2019).

24. A. Avakian et al., "Fractal analysis of region-based vascular change in the normal and non-proliferative diabetic retina," Curr. Eye Res. 24(4), 274-280 (2002).

25. E. V. Costa and R. A. Nogueira, "Fractal, multifractal and lacunarity analysis applied in retinal regions of diabetic patients with and without nonproliferative diabetic retinopathy," Fractal Geom. Nonlinear Anal. Med. Biol. 1(3), 112-119 (2015).

26. N. Popovic et al., "Fractal dimension and lacunarity analysis of retinal microvascular morphology in hypertension and diabetes," Microvasc. Res. 118, 36-43 (2018).

27. S. Tălu, C. Vlăduţiu, and C. A. Lupaşcu, "Characterization of human retinal vessel arborisation in normal and amblyopic eyes using multifractal analysis," Int. J. Ophthalmol. 8(5), 996-1002 (2015).

28. S. Frost et al., "Retinal vascular biomarkers for early detection and monitoring of Alzheimer's disease," Transl. Psychiatry 3, e233 (2013).

29. Y. T. Ong et al., "Retinal vascular fractals and cognitive impairment," Dement. Geriatr. Cognit. Disord. 4(2), 305-313 (2014).

30. M. A. Williams et al., "Retinal microvascular network attenuation in Alzheimer's disease," Alzheimers Dement. 1(2), 229-235 (2015).

31. Ş. Ţălu et al., "Fractal and lacunarity analysis of human retinal vessel arborisation in normal and amblyopic eyes," Hum. Vet. Med. 5(2), 45-51 (2013).

32. C. Y. Cheung et al., "Quantitative and qualitative retinal microvascular characteristics and blood pressure," J. Hypertens. 29(7), 1380-1391 (2011).

33. M. F. Marmor et al., "Standard for clinical electroretinography (2004 update)," Doc. Ophthalmol. 108(2), 107-114 (2004).

34. G. E. Holder et al., "ISCEV standard for clinical pattern electroretinography-2007 update," Doc. Ophthalmol. 114(3), 111-116 (2007).

35. D. C. Hood et al., "ISCEV guidelines for clinical multifocal electroretinography (2007 edition)," Doc. Ophthalmol. 116(1), 1-11 (2008).

36. T. Smith, N. Gildeh, and C. Holmes, "The Montreal cognitive assessment: validity and utility in a memory clinic setting," Can. J. Psychiatry 52(5), 329-332 (2007).

37. Z. S. Nasreddine et al., "The Montreal cognitive assessment, MoCA: a brief screening tool for mild cognitive impairment," J. Am. Geriatr. Soc. 53(4), 695-699 (2005).
38. Ș. Ţălu and S. Giovanzana, "Fractal and multifractal analysis of human retinal vascular network: a review," Hum. Vet. Med. 3(3), 205-212 (2011).

39. A. Karperien, "FracLac for ImageJ—FracLac advanced user's manual," National Institutes of Health, Bethesda, Maryland, 1993, http://rsb.info .nih.gov/ij/plugins/fraclac/fraclac-manual.pdf (accessed 20 April 2019).

40. L. G. França et al., "Fractal and multifractal properties of electrographic recordings of human brain activity: toward its use as a signal feature for machine learning in clinical applications," Front. Physiol. 9, 1767 (2018).

41. K. Shi, C. Q. Liu, and N. S. Ai, "Monofractal and multifractal approaches in investigating temporal variation of air pollution indexes," Fractals 17(4), 513-521 (2009).

42. M. G. Hu, J. F. Wang, and Y. Ge, "Super-resolution reconstruction of remote sensing images using multifractal analysis," Sensors 9(11), 8669-8683 (2009).

43. F. Faul et al., "Statistical power analyses using G*Power 3.1: tests for correlation and regression analyses," Behav. Res. Methods 41(4), 1149-1160 (2009).

44. J. Cohen, "A power primer," Psychol. Bull. 112(1), 155-159 (1992).

45. B. E. O'Bryhim et al., "Association of preclinical Alzheimer disease with optical coherence tomographic angiography findings," JAMA Ophthalmol. 136(11), 1242-1248 2018.

46. N. J. Hart et al., "Ocular indicators of Alzheimer's: exploring disease in the retina," Acta Neuropathol. 132(6), 767-787 (2016).

47. Y. Koronyo et al., "Retinal amyloid pathology and proof-of-concept imaging trial in Alzheimer's disease," JCI Insight 2(16), e93621 (2017).

48. D. Liu et al., "Thinner changes of the retinal nerve fiber layer in patients with mild cognitive impairment and Alzheimer's disease," BMC Neurol. 15(1), 14 (2015).

49. C. Y. Cheung et al., "Retinal ganglion cell analysis using high-definition optical coherence tomography in patients with mild cognitive impairment and Alzheimer's disease," J Alzheimer Dis. 45(1), 45-56 (2015).

50. M. Koronyo-Hamaoui et al., "Identification of amyloid plaques in retinas from Alzheimer's patients and noninvasive in vivo optical imaging of retinal plaques in a mouse model," Neuroimage 54, S204-S217 (2011).

51. M. Prince, R. Bryce, and C. Ferri, "World Alzheimer report 2011: the benefits of early diagnosis and intervention," Alzheimer's Disease International (2011).

52. H. Radhakrishnan and V. J. Srinivasan, "Multiparametric optical coherence tomography imaging of the inner retinal hemodynamic response to visual stimulation," J. Biomed. Opt. 18(8), 086010 (2013).

53. M. Hammer et al., "Retinal venous oxygen saturation increases by flicker light stimulation," Invest. Ophthalmol. Visual Sci. 52(1), 274277 (2011).

54. T. G. Smith, Jr., G. D. Lange, and W. B. Marks, "Fractal methods and results in cellular morphology-dimensions, lacunarity and multifractals," J. Neurosci. Methods 69(2), 123-136 (1996).

Edmund Arthur received his doctor of optometry (OD) degree from Kwame Nkrumah University of Science and Technology-Ghana (2014) and his PhD from Indiana University Bloomington (2018). His $\mathrm{PhD}$ dissertation focused on detecting preclinical retinal changes in diabetic patients using advanced retinal imaging. He is currently a postdoctoral research associate at the Quantitative Imaging and Eye-Brain Research Lab at Bascom Palmer Eye Institute, University of Miami, researching retinal biomarkers for early diagnosis of Alzheimer's disease.

Gabor Mark Somfai received his MD degree (2001) and his PhD degree in ophthalmology (2007) at Semmelweis University in Budapest, Hungary. As an ophthalmologist, his main clinical interest is the medical and surgical therapy of retinal diseases, mostly diabetic retinopathy, while as a researcher, he is focusing on retinal imaging analysis for the understanding of retinal pathophysiology. $\mathrm{He}$ is currently working as a consultant at the Retinology Unit of the Pallas Kliniken, in Olten, Switzerland.

Maja Kostic received her doctor of medicine (MD) degree, masters, and PhD from the University of Novi Sad, Serbia. Her PhD dissertation focused on morphometric fractal analysis of retinal blood vessels development in prematurely born babies. Currently, she is a pediatric ophthalmology fellow at the Bascom Palmer Eye Institute, University 
of Miami. Her research mainly focuses on cognitive functions and retinal biomarkers for the early diagnosis of Alzheimer's disease.

Susel Oropesa received her MD (2018) from Columbia University College of Physicians and Surgeons and her BS degree in molecular biophysics and biochemistry (2014) from Yale University. She is currently an ophthalmology resident at Mount Sinai Hospital. She has broad interests in the field of ophthalmology including retinal and oculoplastic diseases. Her most recent project focuses on discovering patterns in retinal vascular changes that are associated with cognitive impairment.

Carlos Mendoza-Santiesteban received his MD (1994) from Havana Medical University, Cuba. $\mathrm{He}$ is currently an assistant professor of clinical ophthalmology at Bascom Palmer Eye Institute, University of Miami. He has more than 20 years of experience in clinical neuroophthalmology and medical retina with a special interest in diagnostic tools, including visual electrophysiology and retinal imaging. Also, he has extensive experience in conducting comprehensive evaluations in patients with optic neuropathies, familial dysautonomia, and autosomal dominant optic atrophy.

Delia Cabrera DeBuc received her PhD in applied physics (2002) from the University of Michigan. She is currently a research associate professor leading the Quantitative Imaging and Eye-Brain Research Laboratory at the Bascom Palmer Eye Institute, University of Miami. She is a biophysicist with specific training in imaging processing and data science. She is also leading research on ocular imaging biomarkers in CNS neurodegenerative diseases, such as multiple sclerosis, Parkinson's disease, and Alzheimer's disease. 